\title{
Determination of Amino Acid Composition in the Harpagophytum procumbens Root
}

\author{
A.I. Kriukova ${ }^{1}$, I.M. Vladymyrova ${ }^{1}$, O.L. Levashova ${ }^{2}$ and T.S. Tishakova ${ }^{2}$ \\ ${ }^{1}$ National University of Pharmacy, Pushkins'ka St, 53, Kharkiv, Kharkivs'ka oblast, Ukraine, 61000 \\ ${ }^{2}$ Department of Medical and biooganic Chemistry of Kharkiv National Medical University \\ Kharkiv, Ukraine
}

(Received: December 11, 2018; Accepted: April 28, 2019; Published (Web): June 30, 2019)

\begin{abstract}
The amino acid composition of the roots of Harpagophytum procumbens was investigated by the method of high performance liquid chromatography (HPLC) with preliminary derivatization. Sixteen free and thirteen bound amino acids were quantitatively determined. The content of protein-bound amino acids was calculated.
\end{abstract}

Key words: amino acids, Harpagophytum procombens, high performance liquid chromatography.

\section{INTRODUCTION}

Traditional African plant Harpagophytum procumbens (Devil's claw) of the Pedaliaceae family is used to treat joints and spinal disorders, diseases of gastrointestinal tract, liver, kidneys, and bladder for many years. Roots of $H$. procumbens contain a large number of biologically active substances such as: iridoid glycosides (mainly harpagosides, harpagid and procumbide), sugars (tetrasaccharides, stachyose), triterpenoids (oleanolic, ursolic acids), phytosterols ( $\beta$-sitosterol), aromatic acids (caffeic, cinnamic, chlorogenic), flavonoids (luteolin, kaempferol). Iridoid glycosides, which exert antiinflammatory and analgesic effects, are considered to be the main active substances of the plant. ${ }^{1}$

Analysis of the research results showed that the active components of the $H$.procumbens extract suppress the inflammation by reducing the release and synthesis cyclooxygenase (COX) enzyme involved in the inflammatory cascade. The blockade of TNF- $\alpha$, IL-1 $\beta$, and IL- 6 synthesis simultaneously inhibits the production of matrix metalloproteinases in chondrocytes that are involved in degeneration of cartilage tissue. The published results of the previous

Correspondence to: O.L. Levashova

E-mail: olga.jdon78@gmail.com

Dhaka Univ. J. Pharm. Sci. 18(1): 85-91, 2019 (June) DOI: https://doi.org/10.3329/dujps.v18i1.41895 study indicate that the iridoid glycosides (harpagoside and harpagide) shown on figure 1 are highly selective COX-2 inhibitors with the similar binding power to classical non-steroidal antiinflammatory drugs. ${ }^{2,3}$



Figure 1. Iridoid glycosides of devil's claw

Currently, preparations based on the $H$. procumbens extract are considered as a safe and economically justified alternative to chemically synthesized symptom-modifying drugs. ${ }^{4}$

One of the promising directions in modern medicine and pharmacy is a development of co- 
formulated herbal preparation for the treatment and prophylaxis of musculoskeletal diseases. The synergetic effect of active substances allows to provide the necessary pharmacological action and reduce the effective dose of active substances, eliminating the risk of undesirable side effects. Therefore, we developed a co-formulated drug preparation in the form of soft gelatin capsules containing the $H$. procumbens extract and chondroprotectors (glucosamine sulfate and chondroitin sulfate).$^{5,6}$

In this research, we studied the amino acid composition of H. procumbens in order to expand the information about the chemical composition of raw materials and its further use for standardization of raw materials, intermediate products and the resulting dosage form.

Nowadays, liquid chromatography (HPLC) is the most common method for the amino acids determination. ${ }^{7}$ The absence of chromophore groups in most amino acid molecules requires the derivatization stage for their determination using fluorescence detector. The most common methods of HPLC determination of amino acids are cationexchange chromatography with post-column derivatization and reverse-phase chromatography with pre-column derivatization. In pre-column derivatization, o-phthalic aldehyde (OPA), phenylisothiocyanate, dansyl chloride, 9fluorenylmethoxycarbonyl chloride (FMOC) are most often used. ${ }^{8-10}$

\section{MATERIALS AND METHODS}

\section{Preparation of standard solutions}

Amino acid standards (10 to $1 \mathrm{nmol} / \mu \mathrm{l})$.

Solutions of 16 amino acids in five concentrations were collected from Agilent for calibration curves. Each $1 \mathrm{ml}$ ampoule of standards 5061-3334 was divided into $100 \mu \mathrm{l}$ portions in conical vial inserts.

Derivatization reagents. Borate buffers, OPA and FMOC solutions were supplied by Agilent. These reagents were transferred from their container into an autosampler vial.
FMOC reagent. FMOC, contained in a $1 \mathrm{ml}$ ampule, was divided into 10 aliquots $(100 \mu \mathrm{l}$ each). Each aliquot was put into a vial with a vial insert. The vials were capped and stored at $4{ }^{\circ} \mathrm{C}$. Under these storage conditions, the aliquoted solutions are stable for 7-10 days.

OPA reagent. OPA, contained in a $1 \mathrm{~mL}$ ampule, was divided into 10 aliquots (100 $\mu \mathrm{l}$ each). Each aliquot was put into a vial with a vial insert. The vials were capped and stored at $4^{\circ} \mathrm{C}$. Under these storage conditions, the aliquoted solutions are stable for 7-10 days.

\section{Preparation of sample solutions}

Analysis of free amino acids in the roots of $\boldsymbol{H}$. procumbens. $0.148 \mathrm{~g}$ of a powdered raw material (passed through a sieve with a mesh size $355 \mu \mathrm{m}$ ) was placed in a vial, $4 \mathrm{ml}$ of $0.1 \mathrm{~N}$ hydrochloric acid solution was added and kept in an ultrasonic bath at $80^{\circ} \mathrm{C}$ for $3 \mathrm{hrs}$. Then, $0.5 \mathrm{ml}$ aliquot of a centrifuged extract was evaporated on a rotary evaporator, washed three times with distilled water in order to remove hydrochloric acid. The dry residue was resuspended in $0.5 \mathrm{ml}$ of distilled water and filtered through regenerated cellulose (RC) membrane filters (13 mm i.d., $20 \mu \mathrm{l}$ ).

Test solution of total amino acids in the roots of $\boldsymbol{H}$. procumbens. $0.043 \mathrm{~g}$ of a powdered raw material (passed through a sieve with a mesh size 355 $\mu \mathrm{m})$ was placed in a vial, then $1 \mathrm{ml}$ of $6 \mathrm{~N}$ hydrochloric acid solution was added and thermostated at $110^{\circ} \mathrm{C}$. Hydrolysis was carried out for 24 hours. To remove hydrochloric acid, an aliquot of centrifuged hydrolysate was evaporated on a rotary evaporator. The dry residue was resuspended in 0.5 $\mathrm{ml}$ of distilled water and filtered through RCmembrane filters ( $13 \mathrm{~mm}$ i.d., $20 \mu \mathrm{l}$ ).

After that, $0.5 \mathrm{ml}$ of centrifuged hydrolysate was evaporated on a rotary evaporator. The resulting pellet was resuspended in $2.5 \mathrm{ml}$ of distilled water and filtered through RC-membrane filters $(13 \mathrm{~mm}$ i.d., $20 \mu$ l) into vials.

The hydrolyzed samples or solutions of standard amino acid mixture were automatically derivatized 
with OPA and FMOC by programming the autosampler.

Chromatographic experiments were performed on Agilent 1200 (Agilent technologies, USA) liquid chromatograph. Separation of amino acids in the investigated samples was achieved on a Zorbax AAA column $(150 \mathrm{~mm} \times 4.6 \mathrm{~mm} \times 3 \mu \mathrm{m})$. Mobile phase A was 0.040 mole buffer solution of sodium hydrogen phosphate adjusted to $\mathrm{pH} 7.8 \pm 0.05$ with orthophosphoric acid; mobile phase B - acetonitrile : methanol:water (45: $45: 10, \mathrm{v} / \mathrm{v} / \mathrm{v})$. The separation of the amino acids in investigated samples was performed in a gradient elution mode with a constant flow rate $1.5 \mathrm{ml} / \mathrm{min}$. Temperature of the column thermostat was at $40^{\circ} \mathrm{C}$. Excitation (Ex) and emission (Em) wavelength were $340 \mathrm{~nm}$ and $450 \mathrm{~nm}$, correspondingly. Gradient program is shown in table 1.

Identification of amino acids was carried out by comparing the amino acids retention times (RT) in sample with their RT in standards (Agilent 50613334). The content of bound amino acids was determined by subtracting the content of free amino acids from their total content.

Validation of HPLC method for the determination of amino acids. Validation of the above mentioned method was performed according to the following characteristics: specificity, linearity, accuracy, precision, intermediate precision and robustness in a certain range of concentrations.

Table 1. Gradient conditions

\begin{tabular}{cc}
\hline Time $(\min )$ & $\% \mathrm{~B}$ \\
\hline 0.0 & 2 \\
0.35 & 2 \\
13.4 & 57 \\
13.5 & 100 \\
15.7 & 100 \\
15.8 & 2 \\
18.0 & 2 \\
20.0 & end \\
\hline
\end{tabular}

\section{RESULTS AND DISCUSSION}

The specificity study of the present method was performed by a comparison of the retention time (RT) of five different concentrations of the standard mixture of amino acids (Table 2).

As can be seen from table. 2, the relative standard deviation (RSD) for RT values of solutions for the standard sample of the amino acid mixture was from 0.01 to $0.09 \%$. This demonstrates the specificity of the method. The chromatogram of a standard mixture of amino acids is shown in figure 2.

Table 2. The retention time for different concentration of amino acids in standard mixture $(n=5)$

\begin{tabular}{clccccccc}
\hline \multirow{2}{*}{ № } & \multirow{2}{*}{ Amino acids } & \multicolumn{9}{c}{ Concentration, $\mathrm{nmol} / \mathrm{cm}^{-3}$} & \multirow{2}{*}{ Average } & \multirow{2}{*}{ RSD / \% } \\
\cline { 2 - 5 } & & 10 & 25 & 100 & 250 & 1000 & & 0.09 \\
2 & L-Aspartic & 2.528 & 2.529 & 2.528 & 2.531 & 2.534 & 2.530 & 0.05 \\
3 & L-Glutamic & 4.871 & 4.872 & 4.874 & 4.867 & 4.868 & 4.870 & 0.05 \\
4 & L-Serine & 7.358 & 7.354 & 7.363 & 7.361 & 7.363 & 7.360 & 0.03 \\
5 & L-Histidine & 8.206 & 8.208 & 8.202 & 8.204 & 8.209 & 8.206 & 0.05 \\
6 & Glycine & 8.557 & 8.568 & 8.564 & 8.562 & 8.567 & 8.564 & 0.04 \\
7 & L-Threonine & 8.728 & 8.734 & 8.733 & 8.739 & 8.737 & 8.734 & 0.03 \\
8 & L-Alaninine & 9.385 & 9.389 & 9.391 & 9.393 & 9.39 & 9.390 & 0.04 \\
9 & L-Tyrosine & 9.946 & 9.949 & 9.951 & 9.958 & 9.956 & 9.952 & 0.02 \\
10 & L-Valine & 10.997 & 10.992 & 10.994 & 10.996 & 10.998 & 10.995 & 0.02 \\
11 & L-Methionine & 12.887 & 12.889 & 12.883 & 12.887 & 12.881 & 12.885 & 0.02 \\
12 & L-Phenylalanine & 13.039 & 13.036 & 13.038 & 13.031 & 13.033 & 13.035 & 0.02 \\
13 & L-Isoleucine & 14.381 & 14.385 & 14.388 & 14.383 & 14.385 & 14.384 & 0.02 \\
14 & L-Leucine & 14.975 & 14.978 & 14.979 & 14.971 & 14.973 & 14.975 & 0.02 \\
15 & L-Lysine & 15.252 & 15.261 & 15.253 & 15.259 & 15.254 & 15.256 & 0.02 \\
16 & L-Proline & 18.717 & 18.709 & 18.713 & 18.719 & 18.725 & 18.717 & 0.03 \\
\hline
\end{tabular}




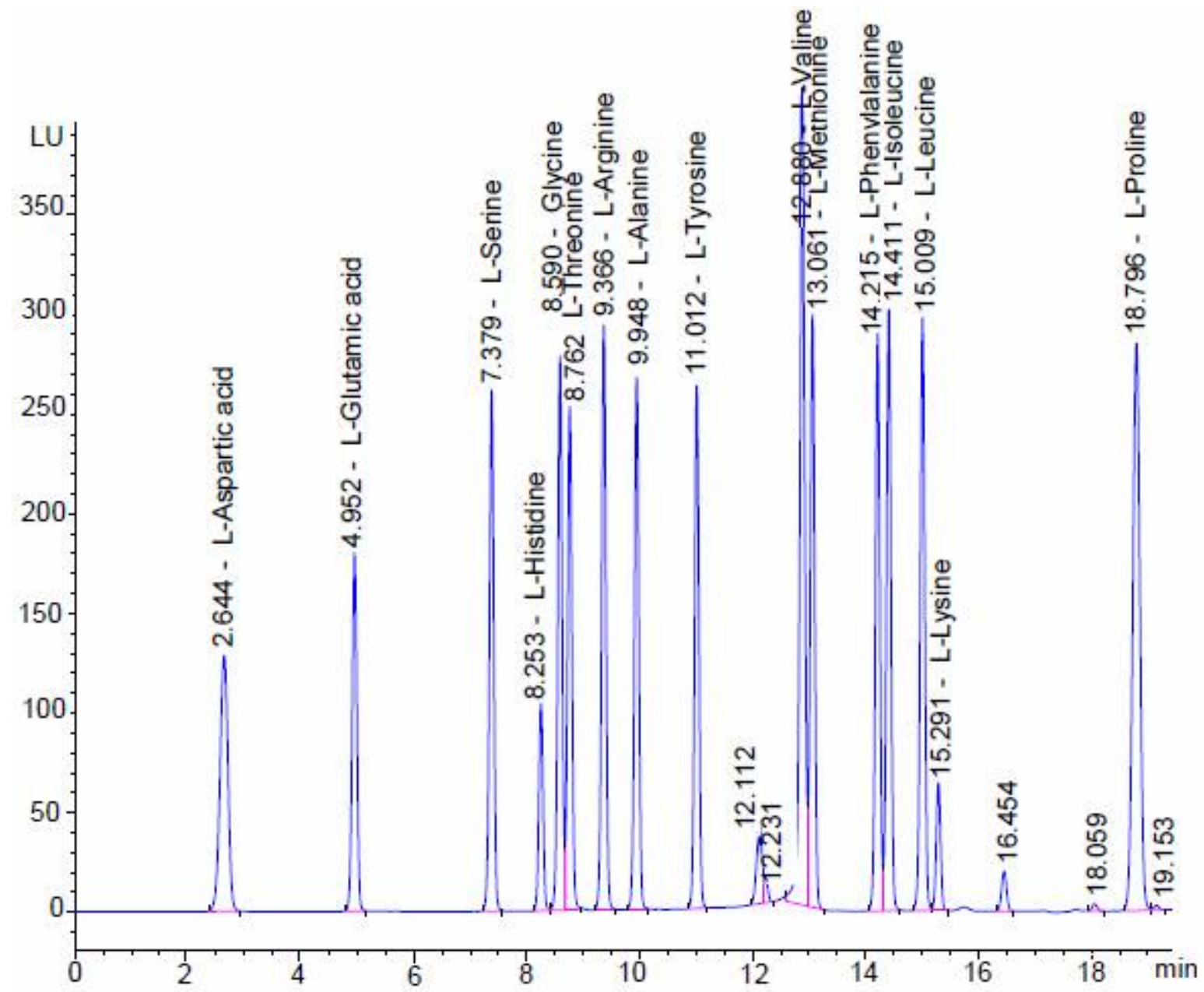

Figure 2. Chromatogram of the standard amino acid mixture.

As a result of the study, 6 free and 13 bound amino acids were identified in the roots of $H$. procumbens. The results of amino acid analysis of the investigated raw material: retention time and quantitative content of amino acids $(\mu \mathrm{g} / \mathrm{mg})$ on dry basis $(\mathrm{n}=5)$ are given in table 3 .

Obtained experimental data indicate that the roots of $H$. procumbens contain a fairly low content of free amino acids $(2.21 \mu \mathrm{g} / \mathrm{mg})$, and main content is represented by bound amino acids $(12.15 \mu \mathrm{g} / \mathrm{mg})$ i.e., the quantitative content of amino acids after total hydrolysis increased by more than 6 times. The highest content is characterized by L-Aspartic, LGlutamic and L-Proline. Amino acids such as LHistidine, L-Arginine and L-Methionine present only in the free state. The chromatogram of the free and total amino acids of the studied sample is shown in figures 3 and 4, respectively.

Biological activity of the identified amino acids is well-known: L-Aspartic acid provides the conversion of carbohydrates into muscle energy. Therefore it is used as additives for athletes during periods of increased physical activity. It also increases the activity of the immune system, raises endurance and retains the ability to work. L-Arginine is a building block of many proteins and actively participates in the regulation of the body metabolism; activates regeneration processes in the post-traumatic period during healing of fractures, healing of trophic ulcers; participates in the processes of collagen 
formation. L-Proline is one of the major amino acids building material of the body, bones, tendons. ${ }^{11-13}$ used for collagen synthesis. Collagen is the main

Table 3. The quantitative composition of amino acid in $H$. procumbens roots $(\mathrm{n}=5)$

\begin{tabular}{clccccc}
\hline \multirow{2}{*}{ No. } & Amino acids & \multicolumn{2}{c}{ Free amino acids } & \multicolumn{2}{c}{ Total amino acids } & $\begin{array}{c}\text { Bound } \\
\text { amino acids }\end{array}$ \\
\cline { 3 - 6 } & & $\begin{array}{c}\text { RT } \\
{[\mathrm{min}]}\end{array}$ & $\begin{array}{c}\text { Amount } \\
{[\mathrm{mkg} / \mathrm{mg}]}\end{array}$ & $\begin{array}{c}\text { RT } \\
{[\mathrm{min}]}\end{array}$ & $\begin{array}{c}\text { Amount } \\
{[\mathrm{mkg} / \mathrm{mg}]}\end{array}$ & Amount $[\mathrm{mkg} / \mathrm{mg}]$ \\
\hline 1 & L-Aspartic & 2.533 & $0.26 \pm 0.01$ & 2.54 & $1.53 \pm 0.01$ & $1.26 \pm 0.04$ \\
2 & L-Glutamic & 4.87 & $0.21 \pm 0.03$ & 4.869 & $2.97 \pm 0.04$ & $2.76 \pm 0.01$ \\
3 & L-Serine & 7.361 & $0.03 \pm 0.01$ & 7.351 & $1.01 \pm 0.03$ & $0.98 \pm 0.03$ \\
4 & L-Histidine & 8.208 & 0.00 & 8.208 & 0.00 & 0.00 \\
5 & Glycine & 8.562 & 0.00 & 8.561 & $0.70 \pm 0.01$ & $0.70 \pm 0.01$ \\
6 & L-Threonine & 8.733 & 0.00 & 8.733 & $0.66 \pm 0.03$ & $0.66 \pm 0.01$ \\
7 & L-Arginine & 9.391 & $1.55 \pm 0.01$ & 9.373 & $1.55 \pm 0.02$ & 0.00 \\
8 & L-Alanine & 9.951 & $0.19 \pm 0.04$ & 9.924 & $0.85 \pm 0.01$ & $0.66 \pm 0.04$ \\
9 & L-Tyrosine & 10.991 & 0.00 & 10.995 & $0.35 \pm 0.01$ & $0.35 \pm 0.01$ \\
10 & L-Valine & 12.88 & $0.03 \pm 0.02$ & 12.853 & $0.74 \pm 0.02$ & $0.71 \pm 0.02$ \\
11 & L-Methionine & 13.031 & 0.00 & 13.031 & 0.00 & 0.00 \\
12 & L-Phenylalanine & 14.184 & 0.00 & 14.191 & $0.71 \pm 0.03$ & $0.71 \pm 0.05$ \\
13 & L-Isoleucine & 14.38 & 0.00 & 14.386 & $0.64 \pm 0.04$ & $0.64 \pm 0.01$ \\
14 & L-Leucine & 14.978 & 0.00 & 14.983 & $0.96 \pm 0.01$ & $0.96 \pm 0.02$ \\
15 & L-Lysine & 15.252 & 0.00 & 15.256 & $0.73 \pm 0.03$ & $0.73 \pm 0.01$ \\
16 & L-Proline & 18.717 & 0.00 & 18.718 & $1.03 \pm 0.01$ & $1.03 \pm 0.04$ \\
Sum of amino acids & & 2.21 & & 14.43 & 12.15 \\
\hline
\end{tabular}

Note - RSD is not more than $2.0 \%$, range for confidence interval: $\pm 0.01-0.5$.

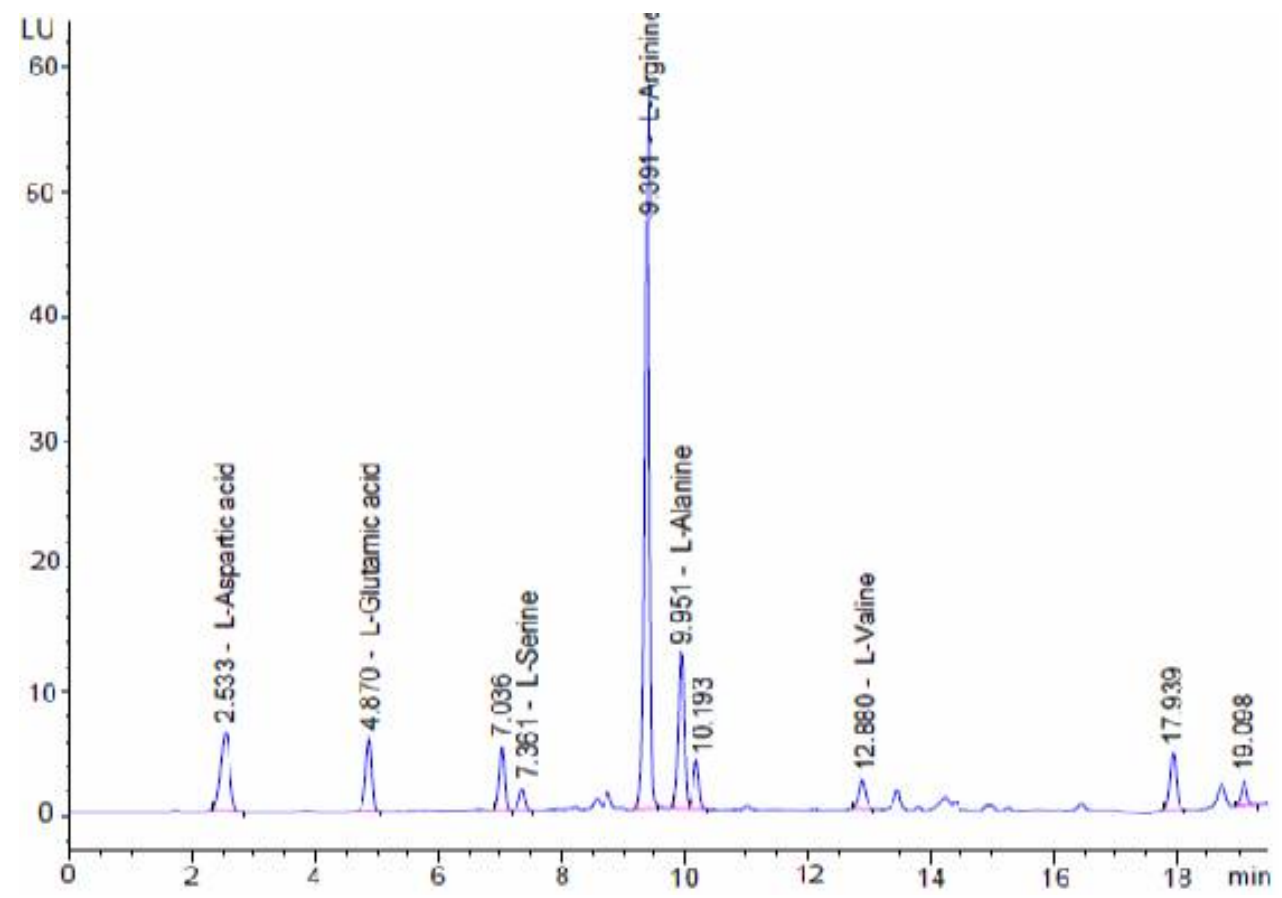

Figure 3. Chromatogram (HPLC) of the free amino acids in the roots of Harpagophytum procumbens. 


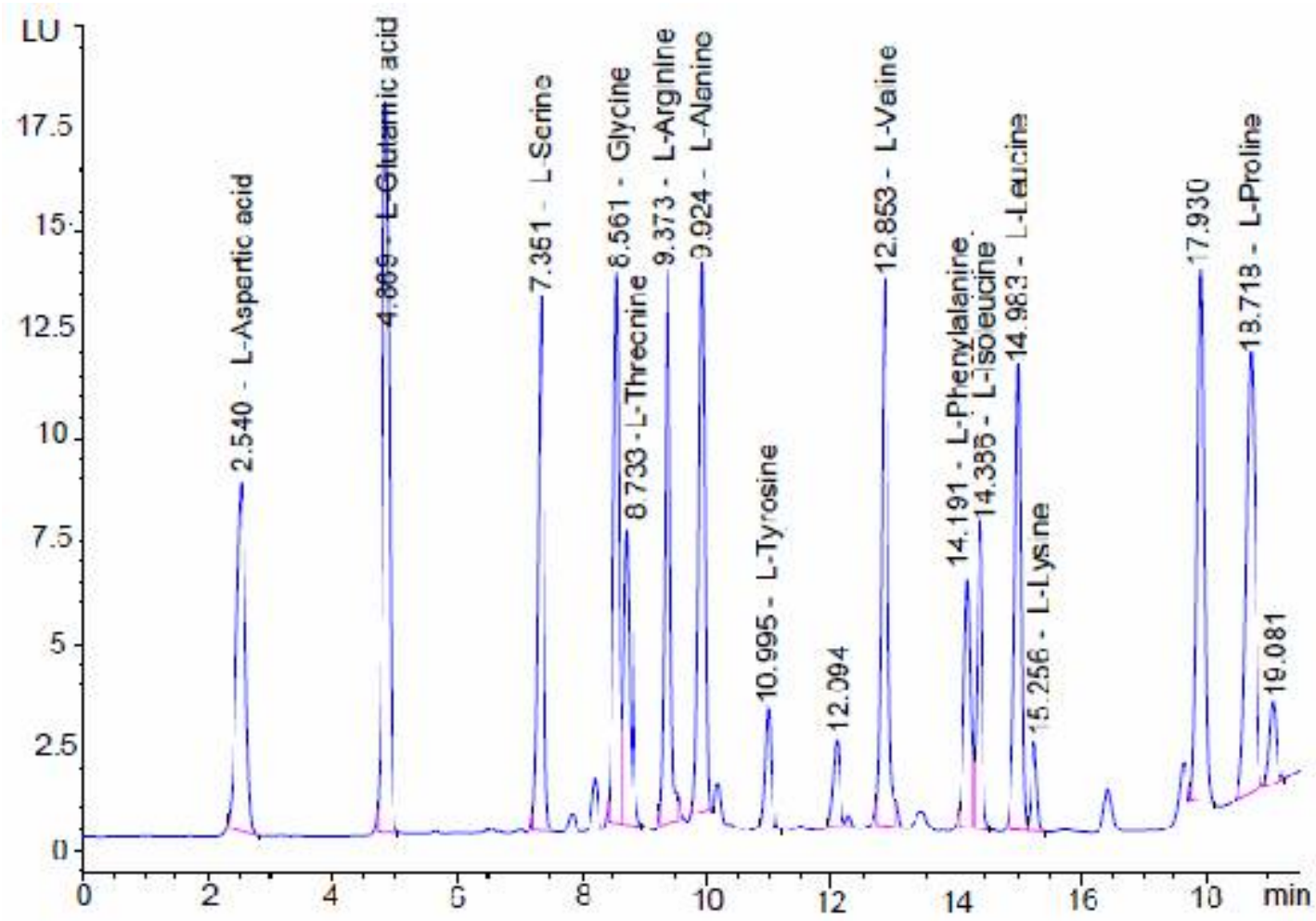

Fig. 4. Chromatogram (HPLC) of the total amino acids in the roots of Harpagophytum procumbens.

\section{CONCLUSIONS}

The qualitative composition and quantitative content of free, bound and total amino acids were determined in the roots of $H$. procumbens using the high-performance liquid chromatography method. 16 free and 13 bound amino acids have been identified. Main components of free amino acids are L-Arginine and L-Aspartic. The major part of the bound amino acids amount is L-Aspartic, L-Glutamic and LProline and their content on dry matter is $1.26,2.76$, $1.03 \mu \mathrm{g} / \mathrm{mg}$, respectively. The quantitative content of amino acids after complete hydrolysis increases 6.5 times.

The obtained results of the determination of amino acid composition in the roots of $H$. procumbens indicate the possibility of application of the investigated raw material as active substance for herbal preparation to treat diseases that are caused by a violation of the collagen synthesis in the human body, in particular arthroses, dermatoses and various forms of periodontal disease.

\section{REFERENCES}

1. Mncwangi, N., Chen, W., Vermaak, I., Viljoen, A.M., Gericke, N. 2012. Devil's Claw - A review of the ethnobotany, phytochemistry and biological activity of Harpagophytum procumbens. J Ethnopharmacol. 143, 755771.

2. Vlachojannis, J., Roufogalis, B. D., Chrubasik S. 2008. Systematic review on the safety of Harpagophytum preparations for osteoarthritic and low back pai. Phytother. Res. 22, 149-152.

3. Fiebich, B.L., Muñoz E., Rose T., Weiss G., McGregor G.P. 2012. Molecular targets of the antiinflammatory Harpagophytum procumbens (devil's claw): inhibition of $\mathrm{TNF} \alpha$ and COX-2 gene expression by preventing activation of AP-1. Phytother. Res. 26, 806-11. doi: 10.1002/ptr.3636

4. Chrubasik, S, Conradt, C, Roufogalis, B.D. 2004. Effectiveness of Harpagophytum extracts and clinical efficacy. Phytother. Res.18, 187-189.

5. Bruyere, O., Cooper, C., Pelletier, J.P. et al. 2014. An algorithm recommendation for the management of knee osteoarthritis in Europe and internationally: a report from a task force of the European Society for clinical and economic aspects of osteoporosis and osteoarthritis (ESCEO). Seminars in Arthritis and Rheumatism. 44, 253-263. 
6. Kriukova, A.I., Vladymyrova, I. M. 2018. The study of the technological and microbiological parameters of a dietary supplement «Osteovert». Visnik Farmaciï. 2, 41-47.

7. Gratzfeld-Huesgen, A. 1999. Sensitive and reliable amino acid analysis in protein hydrolysates using the agilent 1100 Series HPLC; Application note, Agilent Technologies, Inc.Publication number 5968-5658EN

8. Jámbor, A., Molnár-Perl, I. 2009. Amino acid analysis by high-performance liquid chromatography after derivatization with 9-fluorenylmethyloxycarbonyl chloride. Literature overview and further study. J. Chromatogr. A. 1216, 30643077.

9. Jámbor, A., Molnár-Perl, I. 2009. Quantitation of amino acids in plasma by high performance liquid chromatography: Simultaneous deproteinization and derivatization with 9fluorenylmethyloxycarbonyl chloride. J. Chromatogr. A. 1216, 6218-6223.
10. Zhaolai Dai, Zhenlong Wu, Sichao Jia, Guoyao Wu. 2014. Analysis of amino acid composition in proteins of animal tissues and foods as precolumn o-phthaldialdehyde derivatives by HPLC with fluorescence detection. $J$. Chromatography B. 964, 116-127.

11. National Academy of Sciences. 2005. Dietary reference intakes for energy, carbohydrates, fiber, fat, protein and amino acids. Washington, DC, National Academies Press. 1358. doi.org/10.17226/10490

12. Castell, L. 2003. Glutamine supplementation vitro and in vivo, in exercise and in immunodepression. Sports Medicine. 33, 323-45. doi: 10.2165/00007256-200333050-00001.

13. Williams, M. 2005. Dietary supplements and sports performance: Amino acids. J. Int Soc Sports Nutr. 2, 63-7. doi: 10.1186/1550-2783-2-2-63. 